\title{
Fast and Accurate Method for Leaf Area Measurement
}

\author{
Piyush Chaudhary $^{\# 1}$, Sharda Godara ${ }^{* 2}$, A. N. Cheeran ${ }^{\# 3}$, Anand K. Chaudhari ${ }^{\# 4}$ \\ "Electrical Department, Veermata Jijabai Technological Institute, Mumbai, India \\ *Accenture Services Pvt. Ltd., Pune, Maharastra, India
}

\begin{abstract}
Leaf area plays an important role in plant growth analysis and photosynthesis. Traditionally leaf area is measured by regression equitation, grid count method, gravimetric method and planimeter.

In this paper a simple, fast and accurate algorithm for leaf area measurement using image processing is implemented. Image is acquired using digital camera and stored in JPEG format. RGB image is color transformed into CIELAB color space. Color transformed image is segmented using threshold technique. Threshold is calculated using OTSU's method. Holes in leaf region are filled using region filling technique. Number of pixels in square object and leaf region are calculated and leaf area is measured by number of pixels statistics. Accuracy of algorithm is above 99\% which is confirmed by comparing the results of proposed algorithm with grid count method.
\end{abstract}

\section{General Terms}

Digital Image Processing, Image Segmentation.

\section{Keywords}

Image Segmentation, Color Transform, Plant Leaf Spot Disease, Leaf Area Measurement...

\section{INTRODUCTION}

Indian economy is based on agriculture. In total GDP agriculture contributes around $22 \%$ and $65 \%$ of Indian population is directly depend on agriculture [1]. As agriculture make large impact on Indian economy, it is necessary to increase agriculture production. Yield production depends on plant growth which is affected by light, temperature, water and mineral nutrients. Plant growth is depends on leaf area, number of leaf and stem, stem width and plant height.

Leaf area plays an important role in plant growth analysis and photosynthesis. Leaf area is measured using different destructive and non-destructive methods. In destructive methods first the leaf is removed from plant and then measured. In non-destructive methods dimensions of leaf are measured without removing the leaf. Traditionally leaf area is measured by regression equitation, grid count method, gravimetric method and planimeter.

Montgomery (1911) [2] suggested a formula for leaf area measurement which is, $A=b \times l \times w$. Where $\mathrm{b}$ is leaf shape coefficient, 1 is length of leaf and $w$ is width of leaf. This method is non-destructive, quick and easier. But problem is that formula is not uniform for all plants as coefficient $b$ differs among species.

In grid counting method [3] first the leaf is removed from plant and placed on a grid paper. Outlines of the leaf are drawn by pencil on grid paper. Finally leaf area is measured by counting grids covered by leaf. This method is accurate but laborious and time consuming when applied on large number of leaves.

Gravimetric method is also used for leaf area measurement [4]. In this method leaf is first removed from plant and placed on white paper. Paper is cut-out according to the shape of leaf. Now weight of this paper is compared to the weight of known area on the same paper. This method is also laborious and time consuming when applied on large number of leaves.

The planimeter offers a less time-consuming technique, but the precision is limited, especially for relatively small leaves [3].

Some researchers have used image processing techniques for fast and accurate measurement of leaf area [5-8]. Leaf area is measured using following steps: image acquisition, image preprocessing, leaf region segmentation, region filling and area calculation. Some researchers use contour extraction technique $[5,6,7]$ for leaf region segmentation and others use threshold based segmentation [8].

In this paper an image processing based algorithm is implemented for leaf area measurement. The hardware used is a digital camera, a $2 \mathrm{~cm} \times 2 \mathrm{~cm}$ black square, $\mathrm{PC}$ and a white paper sheet. The leaf and square object are placed on white paper sheet and the image is taken using digital camera. Software is implemented in MATLAB 2010a. Experiments were carried upon 70 leaf samples which are different in shape and size.

This paper is organized in four sections. Methodology used is described in section 2 that includes 4 steps as follows, Image acquisition, image color transform, leaf region segmentation and leaf area calculation. Experimentation and results are presented in section 3 . Conclusion to the paper is given in last section.

\section{METHODOLOGY}

Figure 1 shows the flowchart of the steps involved in the leaf area measurement. First images of plant leaf are acquired using digital camera. All the images are stored in JPEG format. To minimize the noise these images are color transformed from RGB image to CIELAB color spaces. Threshold is calculated by applying Otsu method on ' $\mathrm{L}$ ' component of CIELAB color space for image segmentation. Segmented binary image is inverted in color and holes in leaf region are filled using region filling technique. In last leaf area is calculated using known object's area. 


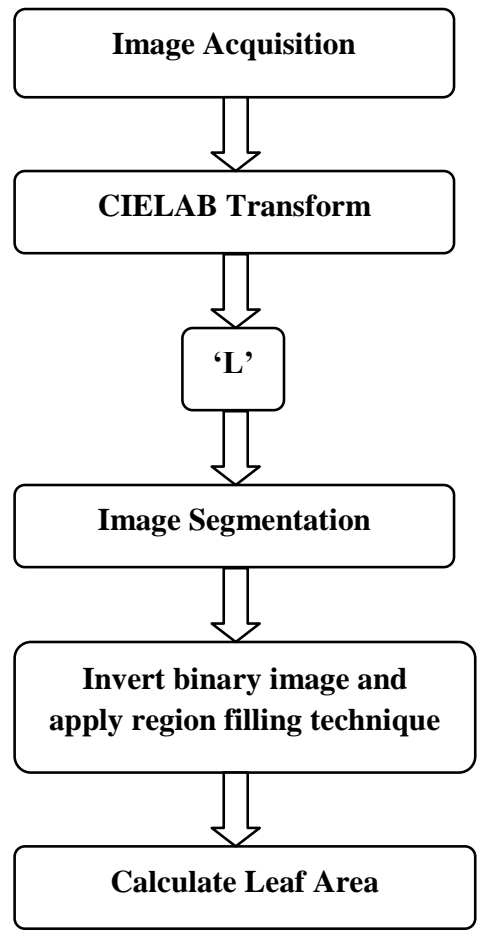

Fig. 1: Flow Chart for Leaf Area Measurement using Image Processing

\subsection{Image Acquisition}

The images of different plant leaves are captured using a CCD color camera (Soney: 16.1 mega pixels). Leaves are collected from field of Agriculture Research Station (A.R.S.) located at Fatehpur Shekhawati, Rajasthan, India. For image acquisition, leaf is placed on white paper sheet with $2 \mathrm{~cm} \times 2 \mathrm{~cm}$ black square as shown in fig 2. All images are stored in JPEG format.

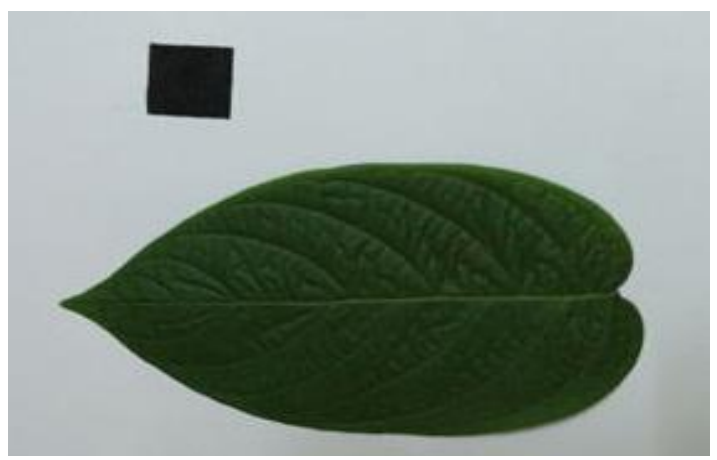

Fig. 2: Image acquisition system

\subsection{Image Color Transform}

To wipe out the noise which is introduced because of background, camera flash and veins RGB image is color transformed into CIELAB color space [9].

CIELAB system is device independent in which brightness and color information is independent of each other. In CIELAB color model brightness information is described by ' $L$ ' component. ' $A$ ' and ' $B$ ' describe color information. Conversion Formulas for LAB color model are [9],

$$
\begin{aligned}
& L=0.2126 * R+0.7152 * G+0.0722 * B \\
& A=1.4749 *(0.2213 * R-0.3390 * G+0.1177 B)+128 \\
& B=0.6245 *(0.1949 * R+0.6057 * G-0.8006 * B)+128
\end{aligned}
$$

\subsection{Leaf Region Segmentation}

Accuracy of leaf area measurement technique is depends on how accurate leaf and known object are detected. In this paper image is segmented using threshold technique. Threshold is applied on ' $L$ ' component of CIELAB color space. Threshold is calculated using OTSU's method [10]. Segmented image is binary image in which leaf and known object are segmented as same color and background is segmented as different color. This segmented image is inverted in color. There may be some cuts and spots on leaf because of diseases and insects. In segmented binary image background and these spots are in same color. Leaf area can't be measured accurately without filling these spots. These holes are filled using region filling technique.

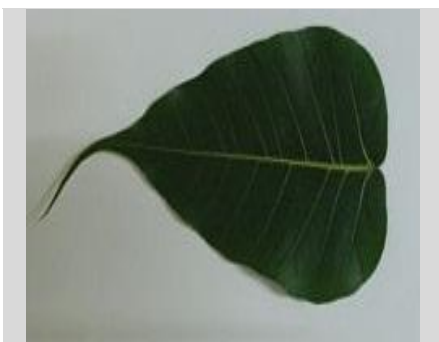

(a)

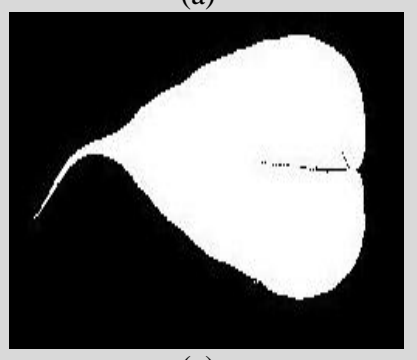

(c)

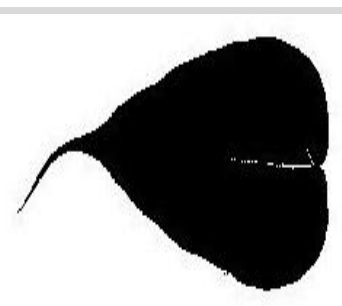

(b)

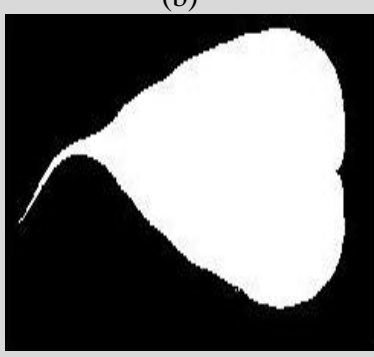

(d)
Fig. 3: RGB image of "Ficus Religiosa” (peepal) leaf (a), segmented image (b), inverted binary image (c), image after applying region filling technique (d).

\subsection{Leaf Area Calculation}

First number of pixels in leaf region and known object region are calculated. In this paper known object is $2 \mathrm{~cm} \times 2 \mathrm{~cm}$ square. So actual area of square object is $\mathbf{4} \mathbf{c m}^{2}$. Now leaf area is calculated using following formula,

$$
A_{l}=\frac{P_{l}}{P_{s}} \times A_{s}
$$

Where $A_{s}$ is area of square object, which is $4 \mathrm{~cm}^{2} . P_{s}$ Is obtained by counting number of pixels in square object and $P_{l}$ is obtained by counting number of pixels in leaf region. Finally leaf area $\left(A_{l}\right)$ is calculated using above formula.

\section{EXPERIMENTATION}

Experiments were carried upon different shaped plant leaves. Area is calculated using proposed algorithm and results are compared with measurements of grid count method. Leaf is first removed from plant and placed on grid paper. Grid size is $1 \mathrm{~mm}^{2}$. Outlines of the leaf are drawn by pencil on grid paper. Finally leaf area is measured by counting grids covered by leaf.

Error in area, measured by proposed algorithm is calculated using formula (5),

$$
\text { Error Percentage }=\left(\frac{A_{g}-A_{l}}{A_{g}}\right) \times 100 \%
$$


Where $A_{l}$ is leaf area, calculated by proposed algorithm and $A_{g}$ is leaf area calculated by grid count method.

Experiments were carried upon 70 leaves of different species. Some of the sample leaves are shown in fig 4 ,

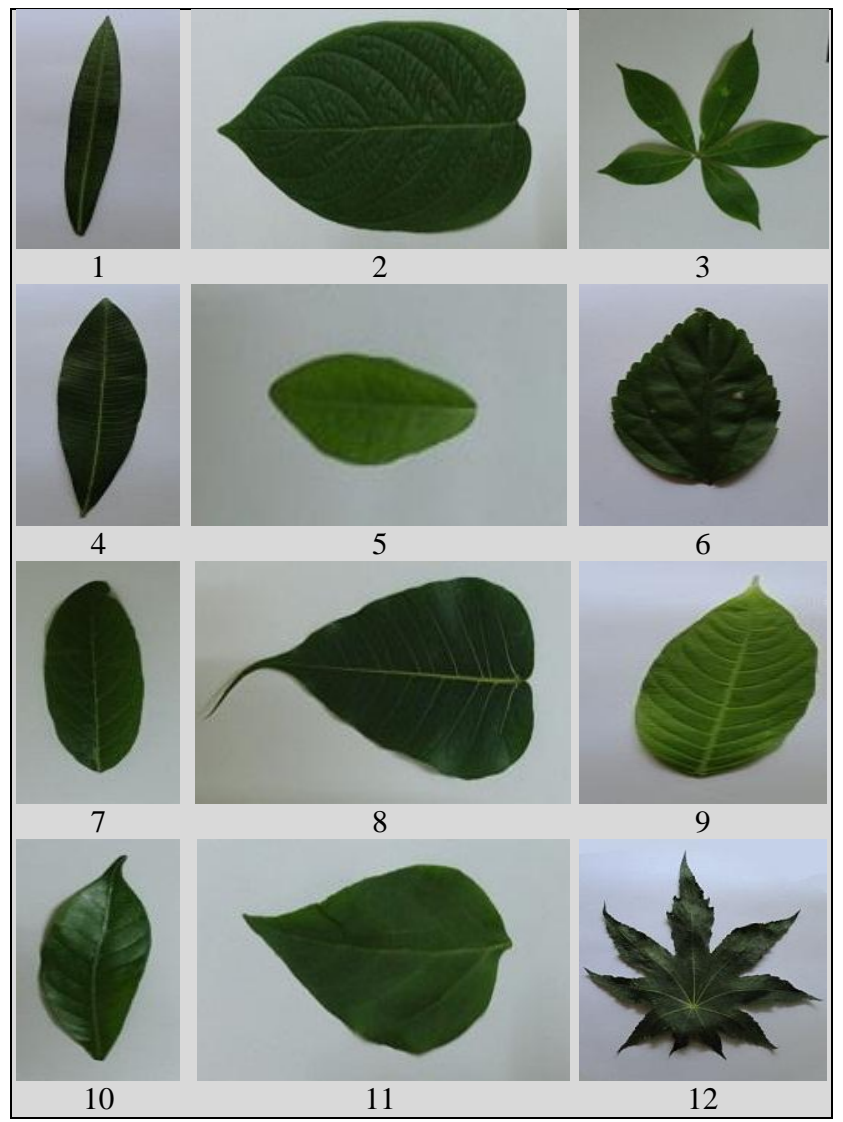

Fig. 4: Sample leaves of different species

Area of above sample leaves is measured using proposed algorithm and grid count method. The results are as given in table 1 .

Table 1: Comparison of results, measured by proposed algorithm and grid count method.

\begin{tabular}{|c|c|c|c|}
\hline Image & $A_{l}\left(\mathrm{~cm}^{2}\right)$ & $A_{g}\left(\mathrm{~cm}^{2}\right)$ & Error \\
\hline 1 & 23.993 & 24.14 & 0.60 \\
\hline 2 & 78.648 & 78.96 & 0.39 \\
\hline 3 & 40.714 & 40.57 & 0.35 \\
\hline 4 & 47.374 & 47.72 & 0.72 \\
\hline 5 & 9.905 & 10.12 & 0.21 \\
\hline 6 & 30.937 & 30.86 & 0.24 \\
\hline 7 & 32.137 & 31.89 & 0.77 \\
\hline 8 & 87.714 & 87.88 & 0.18 \\
\hline 9 & 90.214 & 90.37 & 0.17 \\
\hline 10 & 24.061 & 24.27 & 0.86 \\
\hline 11 & 32.985 & 33.11 & 0.37 \\
\hline 12 & 224.661 & 223.86 & 0.35 \\
\hline
\end{tabular}

Average accuracy of developed algorithm is above $99 \%$ which is confirmed by above experiment.

\section{CONCLUSION}

Leaf area is an important part of plant to analyze the growth and predict the yield. Grid count method and gravimetric method are generally used for leaf area measurement. But these methods are laborious and time consuming when applied on large number of leaves.

An image processing based algorithm is implemented for leaf area measurement. The system requires a digital camera, a PC, $2 \mathrm{~cm} \times 2 \mathrm{~cm}$ black square object and a white sheet. Images are collected in JPEG format. Images are color transformed into CIELAB color space to wipe out the noise. Threshold is calculated on ' $\mathrm{L}$ ' component of CIELAB color space by OTSU's method. Segmented binary image is inverted in color and holes in leaf region are filled using region filling technique. Finally leaf area is measured by pixel number statistic. The results are compared with measurements of grid count method

Experiments were carried upon 70 leaves of different species. Experimental results show that following this method leaf area is measured accurately. Average accuracy of this algorithm is above $99 \%$ which is confirmed by comparing the results with measurements of grid count method.

Future to this it is needed to compute disease severity percentage on plant leaf and integrate both results for actual prediction of yield loss and plant growth

\section{ACKNOWLEDGEMENT}

The authors sincerely like to thank the Agriculture Research Station (A.R.S.), Fatehpur shekhawati (Rajasthan) and VJTI. This work was not possible without help of Dr. S. D. Singh, Dr. Vichitra Singh and all the people who have directly and indirectly encouraged us and helped us in working out our research.

\section{REFERENCES}

[1] "Agriculture overview", available on: http://www.ficcib2b.com/sector-overview-pdf/Sector-agri.pdf, accessed on may 24, 2012.

[2] Montgomery EG," Correlation studies in corn. Nebraska", Agr Exp Sta Annu Rep 24, pp.108-159, 1911.

[3] Linda Styer Caldas, Carlos Bravo, Homero Piccolo, Carmen Rachel S. M. Faria," Measurement of Leaf Area With a Hand-Scanner Linked to a Microcomputer", R. Bras. Fisiol. Veg. 4(1), pp. 17-20.

[4] Ross, J., Ross, V., " Phytometrical characteristics of the willow plantation at Toravere", joint Swedish-estonian seminar, 24-26 september 1995. swedish univ. agric. sci. uppsala, report 57, pp. 133-145, 1995

[5] Chaohui Lü, Hui Ren, Yibin Zhang, Yinhua Shen," Leaf Area Measurement Based on Image Processing", IEEE International Conference on Measuring Technology and Mechatronics Automation, pp. 580-582, 2010.

[6] Hassan Hajjdiab, Abdellatif Obaid," A Vision-based Approach for Nondestructive Leaf Area estimation", 2nd Conference on Environmental Science and Information Application Technology, IEEE, pp.53-56, 2010.

[7] Tan Feng, Wang Chun, " Calculating the Leaf-Area Based on Non-Loss Correction Algorithm", International Conference of Information Science and Management Engineering, IEEE, pp.75-78, 2010. 
[8] Sanjay B. Patil, Dr. Shrikant K. Bodhe," Betel Leaf Area Measurement Using Image Processing", International Journal on Computer Science and Engineering (IJCSE), Vol. 3, pp. 2656-2660, 7 July 2011

[9] Piyush Chaudhary, Anand K. Chaudhari, Dr. A. N. Cheeran and Sharda Godara, "Color Transform Based
Approach for Disease Spot Detection on Plant Leaf", International Journal of Computer Science and Telecommunications (IJCST), Vol. 3, Issue 6, Jun 2012.

[10] Nobuyuki Otsu, "A Tlreshold Selection Method from Gray-Level Histograms", IEEE transactions on systrems, man, and cybernetics, VOL. SMC-9, no. 1, january 1979 